\title{
Dry contact electrodes performance in canine ECG
}

\author{
Juhani Virtanen \\ Joni Leivo \\ Antti Vehkaoja \\ BioMediTech Institute \\ and Faculty of \\ Biomedical Sciences \\ and Engineering, \\ Tampere University of \\ Tampere, Finland
}

\author{
Sanni Somppi \\ Heini Törnqvist \\ Department of Equine \\ and Small Animal \\ Medicine, University \\ of Helsinki \\ Helsinki, Finland \\ Patrique Fiedler \\ Institute of Biomedical \\ Engineering and \\ Informatics, \\ Technische Universität \\ Ilmenau, \\ Ilmenau, Germany
}

\author{
Heli Väätäjä \\ Veikko Surakka \\ Research Group for \\ Emotions, Sociality, \\ and Computing, \\ Faculty of \\ Communication \\ Sciences, University of \\ Tampere (UTA), \\ Tampere, Finland
}

\begin{abstract}
Measuring the heart rate of animals is an important area of research which can be applied to numerous analyses. In this work we evaluate the performance of two dry contact electrocardiogram (ECG) electrode structures for monitoring the heart rate of domestic pets. The aim was to improve the operation of previously evaluated electrodes with some modifications. First, the thirty-pins of a previously studied polymer electrode with a silver/silver chloride $(\mathrm{Ag} / \mathrm{AgCl})$ coating were reduced to 12 . Second, a 12-pin gold-plated metal electrode was coated with poly $(3,4$ ethylenedioxythiophene) polystyrene sulfonate (PEDOT:PSS). These electrodes were attached to a specifically designed measurement harness and the performance of the electrodes was evaluated in terms of heartbeat detection coverage. The heart rate coverage was measured during four different behaviors: standing, sitting, lying, and walking ( $\mathrm{N}=27 /$ activity). The results were, in general, comparable to the previously reported performance but having fewer pins on a polymer electrode seemed to cause more variation in the coverage values. However, when measures such as the median value of the coverages are considered, there was no obvious difference, especially when the coverage values were observed altogether. Thus, new electrode solutions are worthy of further research.
\end{abstract}

\section{Author Keywords}

Canine; Electrocardiogram; Dry Electrode

\section{ACM Classification Keywords}

[Hardware; Communication hardware, interfaces and storage; Sensors and actuators];

\footnotetext{
Permission to make digital or hard copies of part or all of this work for personal or classroom use is granted without fee provided that copies are not made or distributed for profit or commercial advantage and that copies bear this notice and the full citation on the first page. Copyrights for thirdparty components of this work must be honored. For all other uses, contact the Owner/Author.
}

ACI18, December 4-6, 2018, Atlanta, GA, USA (C) 2018 Copyright is held by the owner/author(s). ACM ISBN 978-1-4503-6219-1/18/12. https://doi.org/10.1145/3295598.3295609

\section{INTRODUCTION}

Heart rate monitoring is increasingly popular with pets; the motivation being to obtain information about the physical and emotional well-being of the animal of which pet-owners are always interested in $[1,4]$. There are some currentlyavailable commercial devices for monitoring the heart rate of animals in their everyday lives. However, all such devices are limited by the fact that the information is not always reliable, or if the devices are able to differentiate and extract reliable heart rate information, the coverage of the readings is low so no heart rate is usually obtained during movement. Furthermore, there is no way to perform electrocardiogram (ECG) monitoring with the currently available devices. There are various reasons for this, one of the most obvious ones being that the available devices often require wet-gel patch electrodes which entail shaving the animal's fur to get a sufficiently high-quality electrical contact with the skin. Shaving the skin and applying conductive gel for the everyday monitoring of a pet has always been problematic, so the need for low-maintenance electrodes is clear. One possible approach to this problem is to use dry electrodes. Although promising results have been reported for both human and animal applications [2,5,6], there are challenges in measuring ECG with dry electrodes. These are mainly associated with disturbances caused by motion artefacts, often resulting in the total loss of electrical contact between the electrode and the skin. These phenomena are particularly apparent in so-called dynamic activity modes, such as walking of the dog.

The performance of our ECG electrodes was assessed using a previously published methodology [6] in which the performance of three dry ECG electrodes was evaluated in terms of ECG coverage ratio. The coverage ratio was defined as the ratio between the time when the $\mathrm{R}$ - peaks were successfully recovered against the total testing time. The coverage ratio value '1' represents $100 \%$ recovery of the Rpeaks and the value ' 0 ' represents $0 \% \mathrm{R}$ - peak detection. [6]. Thus, the methodology and evaluation criteria applied in this study are the same as the ones presented in the previous study [6]. Both of the electrodes evaluated in this study are modifications or improved versions of the ones presented in 
the previous study. Our aim was to see whether reducing the number of pins on the polymer electrode, or coating the metal pin electrode with a PEDOT:PSS would improve the coverage rate.

\section{MATERIALS AND METHODS}

\section{Electrodes}

Two types of electrode were constructed. The first one was modified from the above-mentioned Ag/AgCl-coated polymer pin electrode manufactured by the University of Ilmenau, Germany. The electrode pin distribution was made less dense by using fewer pins. The hypothesis here was to improve the performance of the electrode by improving its penetration through the animal's fur and simultaneously increasing the applied surface pressure. The second electrode was a modification of the gold-plated metal pin electrode used in the previous study. This electrode was modified by giving it a PEDOT:PSS coating to enhance the electrochemical performance at the electrode/skin interface.

\section{The polymer electrode}

The silver/silver chloride- $(\mathrm{Ag} / \mathrm{AgCl})$-coated polymer electrode [3] was prepared for the measurements by removing $60 \%$ of the pins in the original design. Therefore, 18 pins from of the original 30-pin electrode were cut out, giving us a 12-pin polymer electrode for the tests. The trick was to remove the pins so that the remaining pins would form as evenly distributed a pattern as possible. There were two reasons for this. One was to observe the effect of having fewer pins, and the other was to observe the effect of the higher surface pressure of the electrode in the skin contact. The modified electrode and the electrode used in the silicone rubber casting are presented in Figures 1. a) and 2. a) respectively. This electrode is henceforth referred to as the Polymer12 electrode.

\section{Spring-loaded electrode with a PEDOT:PSS coating}

The PEDOT:PSS electrode was prepared by dipping the tips of a spring-loaded gold-plated electrode into a $2.5 \mathrm{wt}$. \% PEDOT:PSS/deionised water solution for $1 \mathrm{~h}$ in order to enhance the electrochemical interface at the skin-electrode interface. The mechanical design of this particular electrode was identical to the one studied previously in [6]. Both the electrode itself, and the electrode embedded in the silicone rubber casting are shown in Figures 1. b) and 2. b) respectively. This electrode is henceforth referred to as the PEDOT -electrode.

\section{The fixtures for the electrodes}

The silicone rubber fixtures were prepared by molding a lump of silicone rubber into a 3D -printed mold. The fixtures were used to house the electrodes and make it easier to attach the electrode-fixture to the harness. The electrodes and fixtures were joined together using a second silicone rubber molding in which the fixtures became integral electrodefixture structures. These fixtures were then attached to a harness, which was put on the test animal. The way the silicone rubber fixtures were made is described in detail in [6].
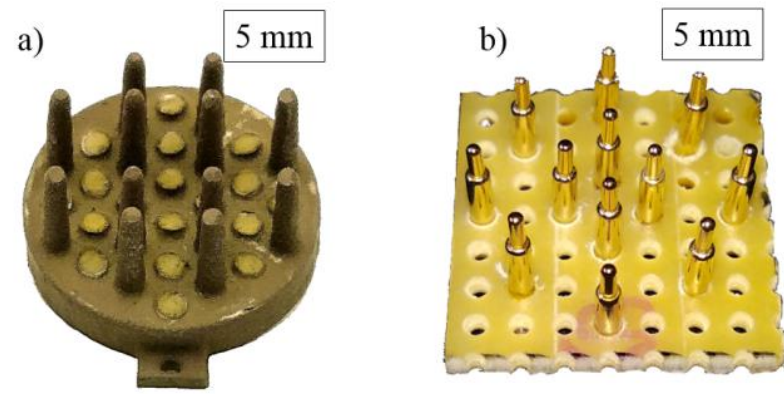

Figure 1. Electrodes a) Reduced pin count polymer (Polymer12) electrode b) PEDOT:PSS coated (PEDOT).electrode
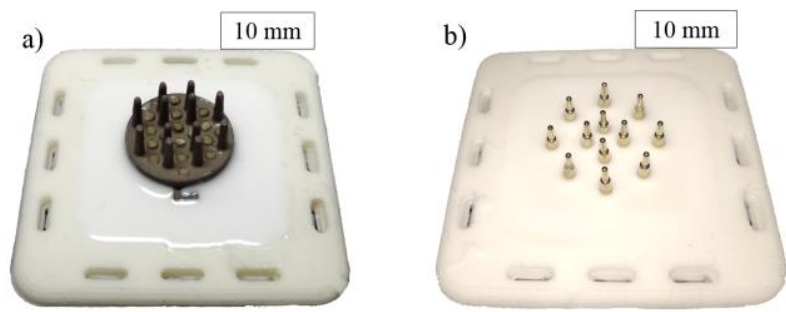

Figure 2. Electrodes in the silicone cast housings a) Reduced pin count polymer (Polymer12) electrode b) PEDOT:PSS coated (PEDOT) electrode in the housings.

\section{The Harness}

The electrode fixtures, comprising a total of four electrode fixtures, were attached to the harness in order to fix the locations of the measurements on the dog. This is illustrated in Figure 3, where the dog, a Golden retriever, is wearing the harness containing the electrode fixtures. The electrode locations in the harness are marked with circles.

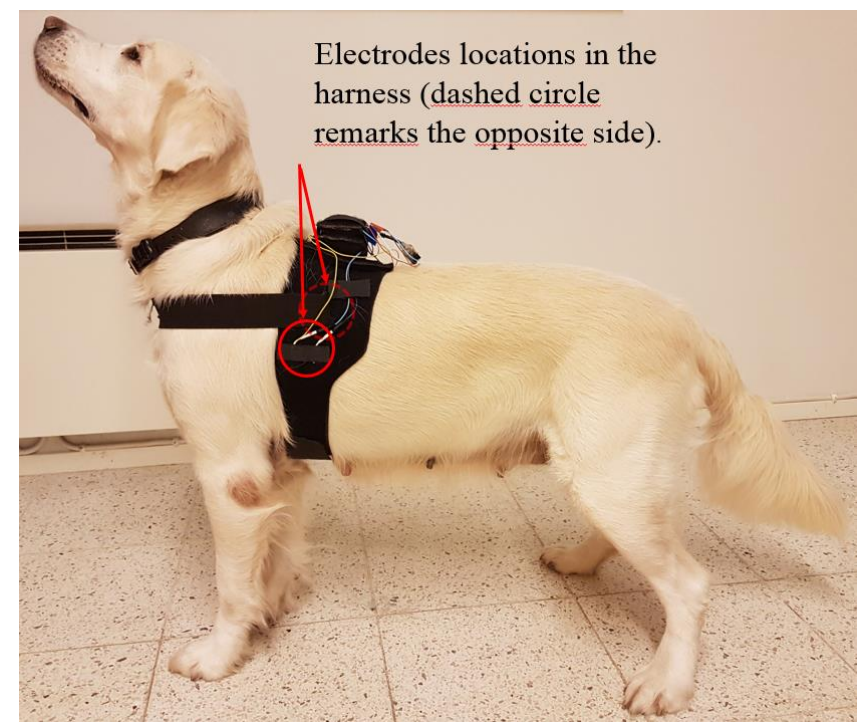

Figure 3. The dog, a Golden retriever, and harness with electrode locations. The solid circle represents the left-hand electrode while the dashed circle is the right-hand electrode. 


\section{Testing procedure}

All the tests and measurements were conducted by the University of Helsinki according to statement 2/2018 of the Ethical Committee for the Use of Animals in Experiments. The three dogs that were chosen to participate in the measurement sessions, two female Beauce Shepherds and one male Hovawart, were the same dogs that had been used in the previous study [6]. The testing procedure involved four activity modes: standing, sitting, lying and walking. Each of the activities were measured for one minute making the length of each 'testing block' 4 minutes. The blocks were then repeated three times, making a total of 12 minutes for each test session. The test sessions were repeated three times, and the harness was removed between each session. All of the electrodes were tested in the same way, giving a total number of one-minute activities $(\mathrm{N}=9)$ in each configuration. The total recording time in the test was therefore 216 minutes, representing 2161 -minute activity-mode samples.

\section{The measurement electronics}

A custom-made physiological measuring apparatus, the SpiritCor9D monitoring device, was used to record the ECG biopotential data at a sampling frequency of $500 \mathrm{~Hz}$. The measurement device was exactly the same as the one used in the previous work except that it had a higher sampling frequency. This was taken into account later in the dataprocessing stage to ensure comparability with the previous measurements. In order to collect the measurement data, the electrodes in the harness were physically connected to the measurement device by wire. The recorded ECG data was later processed and analyzed with a standard desktop home computer .

\section{Data analysis}

A digital Butterworth band pass filter $(2-30 \mathrm{~Hz})$ was applied to the raw ECG measurement data. This filtering was done to remove power line noise, baseline wander and motion artefacts from the measured ECG signal. After this filtering, a pattern-matching algorithm was applied to the filtered signal which matched a predefined ECG template, and could therefore extract the R-peaks. The coverage ratios were then computed from this R-peak detection data. This procedure is described in more detail in [6].

\section{RESULTS}

The measurement results are shown in Tables 1 to 3 and visualized in the box plots in Figures 4 and 5. The tables present the results in statistical terms of average, mean, range and standard deviation in both their consolidated forms (Table 1) and broken down into the individual measurements taken from each dog (Tables 2-3). Moreover, the coverage of each activity mode is also listed separately in the tables. Figure 4 shows the total coverage boxplots of the two electrodes, while Figure 5 shows the boxplots broken down so that they represent the measurements of each dog separately. The average, the standard deviation, the median and the range values of the overall coverage varied between $0.15-0.81,0.08-0.37,0.14-0.80$ and $0.21-0.34$ respectively. These values are shown in Table 1 . In the individual test case break-downs, the coverage average, standard deviation, median and range values with the Polymer12 and the PEDOT are 0.29-0.83, 0.15-0.34, 0.25-0.94, 0.39-0.97 (Table 2); 0.13-0.96, 0.08-0.37, 0.03-0.99, 0.21-0.97 (Table 3.) for the two electrodes respectively.

\begin{tabular}{|c|c|c|c|c|c|c|c|c|c|c|c|c|c|c|c|c|}
\hline \multirow{2}{*}{ Overall } & \multicolumn{4}{|c|}{ Stand } & \multicolumn{4}{|c|}{ Sit } & \multicolumn{4}{|c|}{ Lie } & \multicolumn{4}{|c|}{ Walk } \\
\hline & Avg & Sd & Md & $\mathbf{R}$ & Avg & Sd & Md & $\mathbf{R}$ & Avg & Sd & Md & $\mathbf{R}$ & Avg & Sd & Md & $\mathbf{R}$ \\
\hline Polymer12 & 0.78 & 0.19 & 0.72 & 0.28 & 0.69 & 0.30 & 0.41 & 0.24 & 0.81 & 0.12 & 0.77 & 0.34 & 0.15 & 0.08 & 0.14 & 0.21 \\
\hline PEDOT & 0.78 & 0.21 & 0.80 & 0.25 & 0.45 & 0.37 & 0.47 & 0.30 & 0.81 & 0.12 & 0.77 & 0.34 & 0.15 & 0.08 & 0.14 & 0.21 \\
\hline
\end{tabular}

Table 1. Overall coverages obtained with both electrodes. $\mathrm{Avg}=$ average, $\mathrm{Sd}=$ standard deviation, $\mathrm{Md}=\mathbf{m e d i a n}, \mathbf{R}=\mathbf{r a n g e}$

\begin{tabular}{ccccccccccccccccc} 
Polymer12 & \multicolumn{4}{c}{ Stand } & \multicolumn{4}{c}{ Sit } & \multicolumn{4}{c}{ Lie } & \multicolumn{4}{c}{ Walk } \\
& Avg & Sd & Md & R & Avg & Sd & Md & R & Avg & Sd & Md & R & Avg & Sd & Md & R \\
\hline Dog1 & 0.78 & 0.20 & 0.83 & 0.63 & 0.65 & 0.34 & 0.71 & 0.97 & 0.83 & 0.21 & 0.94 & 0.53 & 0.50 & 0.26 & 0.59 & 0.66 \\
$\operatorname{Dog} 2$ & 0.83 & 0.15 & 0.83 & 0.39 & 0.82 & 0.16 & 0.83 & 0.41 & 0.60 & 0.34 & 0.57 & 0.93 & 0.43 & 0.27 & 0.50 & 0.66 \\
$\operatorname{Dog} 3$ & 0.73 & 0.20 & 0.69 & 0.60 & 0.69 & 0.30 & 0.82 & 0.80 & 0.63 & 0.32 & 0.69 & 0.83 & 0.29 & 0.17 & 0.25 & 0.46
\end{tabular}

Table 2. Individual coverages of Polymer12 electrode. Avg = average, $\mathrm{Sd}=$ standard deviation, $\mathrm{Md}=$ median, $\mathrm{R}=\mathbf{r a n g e}$

\begin{tabular}{|c|c|c|c|c|c|c|c|c|c|c|c|c|c|c|c|c|}
\hline \multirow[t]{2}{*}{ PEDOT } & \multicolumn{4}{|c|}{ Stand } & \multicolumn{4}{|c|}{ Sit } & \multicolumn{4}{|c|}{ Lie } & \multicolumn{4}{|c|}{ Walk } \\
\hline & Avg & Sd & Md & $\mathbf{R}$ & Avg & Sd & Md & $\mathbf{R}$ & Avg & Sd & Md & $\mathbf{R}$ & Avg & Sd & Md & $\mathbf{R}$ \\
\hline Dog1 & 0.76 & 0.17 & 0.69 & 0.41 & 0.68 & 0.37 & 0.76 & 0.97 & 0.42 & 0.36 & 0.47 & 0.92 & 0.50 & 0.26 & 0.59 & 0.65 \\
\hline Dog2 & 0.90 & 0.19 & 0.99 & 0.59 & 0.96 & 0.08 & 0.99 & 0.24 & 0.13 & 0.18 & 0.03 & 0.44 & 0.75 & 0.12 & 0.79 & 0.39 \\
\hline Dog3 & 0.70 & 0.23 & 0.79 & 0.60 & 0.76 & 0.15 & 0.77 & 0.48 & 0.81 & 0.12 & 0.77 & 0.34 & 0.15 & 0.08 & 0.14 & 0.21 \\
\hline
\end{tabular}

Table 3. Individual coverages of PEDOT coated electrode. $\mathrm{Avg}=$ average, $\mathrm{Sd}=$ standard deviation, $\mathrm{Md}=\mathbf{m e d i a n}, \mathbf{R}=\mathbf{r a n g e}$ 

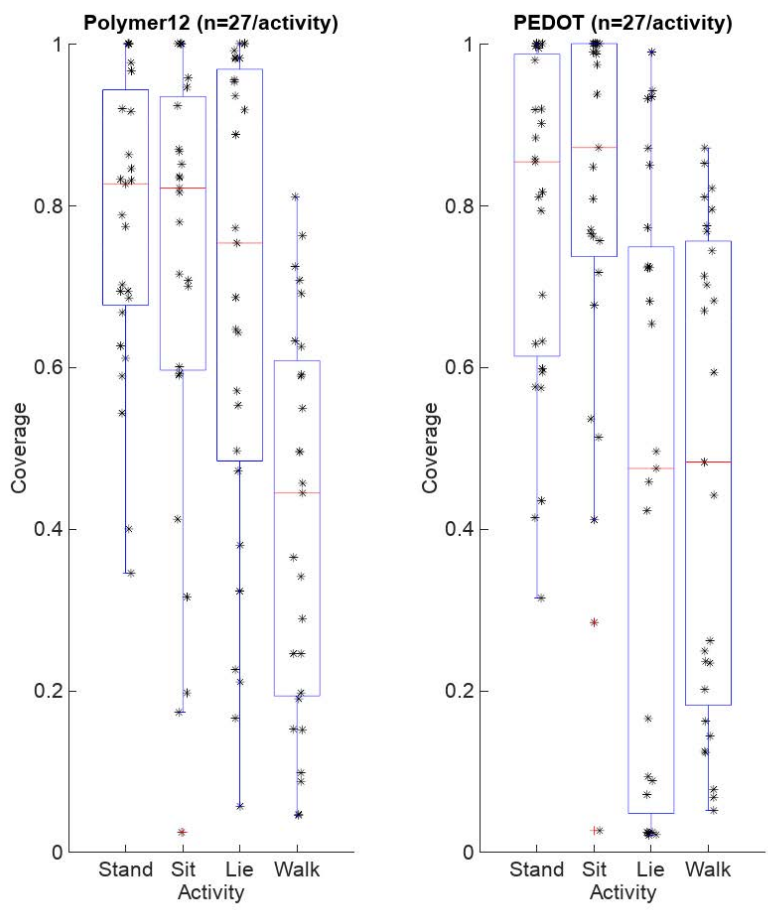

Figure 4. Overall coverage boxplots of the two electrodes in different activities. A star represents an individual coverage observation.
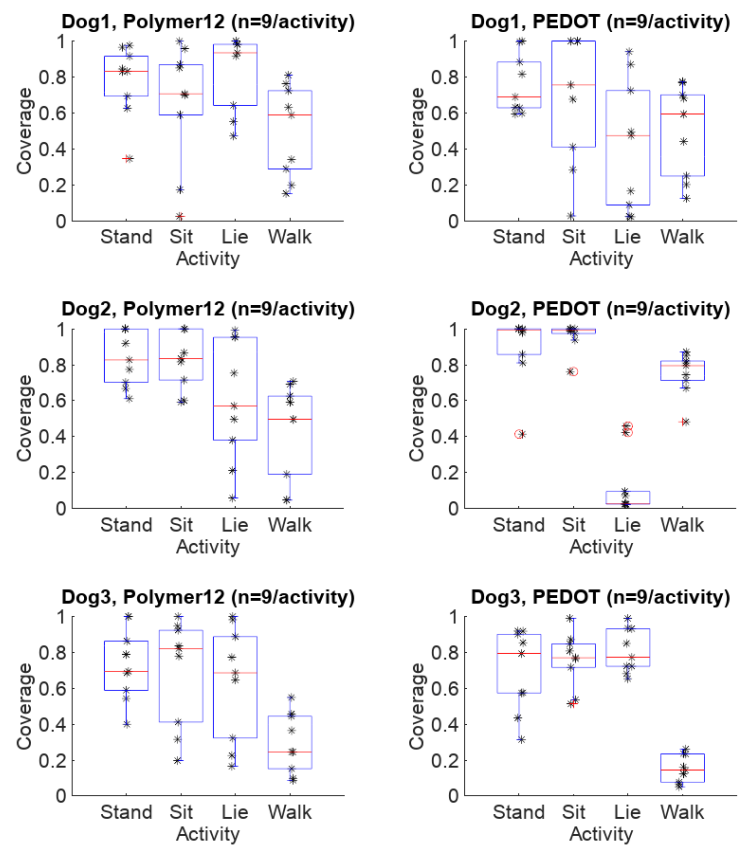

Figure 5. Coverages boxplots of the different electrodes for each measured dog. A star represents an individual coverage observation while a red circle represents an outlier.

\section{CONCLUSIONS}

The results of this study show that the canine heart rate coverage measurements are similar to those reported previously in [6]. As expected, the dry electrodes tend to perform more reliably in stationary postures such as stand and sit. The sitting position is particularly favorable and consistently yields high coverage ratios. As the study was not particularly extensive in that the measurement data was only collected from three dogs, the results should be interpreted as indicative, rather than conclusive. However, the results do indicate that this topic is worthy of further study.

The measurement results give a deeper insight into the effect of having fewer pins on a cast polymer electrode as this seems to cause greater variation in the coverage values. This conclusion is supported by observing the individual electrode canine test-case results. However, if statistical measures such as the median value of the computed coverages are considered, the difference is not particularly great, especially when the coverage values are taken as a whole. Another conclusion is that because the results from the PEDOT:PSS coated electrode are very similar to those from the plain gold-plated electrodes reported earlier, there is no support for the hypothesis that a PEDOT:PSS coating improves the skin-electrode interface. Nevertheless, there still remains the fact that the coverage values are lower than expected in the lying posture. This may have been caused by a change in the electrode-harness attachment during the test as is indicated in one particular test-case (Dog2/PEDOT). Additionally, in this test-case the coverages for walking, standing and sitting are high. This anomaly also motivates further study of this particular phenomenon as dynamic activity modes such as walking have previously been reported as being more challenging. Another possible reason for the differences in the results of this study compared to the previous one [6] may be variations in the testing procedure. Although the tests followed the same protocol and the dogs and testers were the same, which should enable a direct comparison of the results, it is difficult to standardize the behavior of the dogs in absolute terms, particularly when they are walking.

Although the durability of the devices and the effects of longterm wear were not specifically studied, it seems safe to assume that the thin coatings on the electrodes will have a limited lifetime, especially in high-activity operation. Therefore. if these kinds of coatings were to be used for the consumer market, further studies would be required to investigate the long-term durability of the electrodes, which could perhaps be optimized via the coatings and their manufacturing processes. Further studies are also needed to determine how the wear of the electrode influences the quality of the coverage ratio.

Another important aspect of wearable electrodes not covered in this study is the comfort of the electrodes, especially for long-term use. Although the authors did not notice any abnormal behavior from the dogs when they (the dogs) were 
wearing the harness, and the comfort of the electrodes was also declared not to be too irritating by the authors, it is difficult to know what the animals felt about wearing the harness. Despite the importance of this topic, there are currently no objective methods for measuring the degree of comfort or discomfort felt by animal test-subjects.

\section{ACKNOWLEDGMENTS}

This research was funded by Business Finland, a Finnish national research funding organization, grant numbers $1665 / 31 / 2016,1894 / 31 / 2016,7244 / 31 / 2016$ in the context of "Buddy and the Smiths 2.0" project.

\section{REFERENCES}

1. Abbott, Jonathan A. "Heart rate and heart rate variability of healthy cats in home and hospital environments." Journal of feline medicine and surgery 7.3 (2005): 195-202.

2. Brugarolas, Rita, et al. "Wearable heart rate sensor systems for wireless canine health monitoring." IEEE Sensors Journal 16.10 (2016): 3454-3464

3. Fiedler, Patrique, et al. "Contact Pressure and Flexibility of Multipin Dry EEG Electrodes." IEEE Transactions on Neural Systems and Rehabilitation Engineering (2018).

4. Katayama, Maki, et al. "Heart rate variability predicts the emotional state in dogs." Behavioural processes 128 (2016): 108-112.

5. Salvo, Pietro, et al. "A 3D printed dry electrode for ECG/EEG recording." Sensors and Actuators A: Physical 174 (2012): 96-102.

6. Virtanen, Juhani, et al. "Evaluation of dry electrodes in canine heart rate monitoring." Sensors (Basel, Switzerland) 18.6 (2018). 